\title{
The Screensaver Map: Dynamics on Elliptic Curves Arising from Polygonal Folding*
}

\author{
J. Esch and T. D. Rogers \\ Department of Mathematical Sciences, University of Alberta, \\ Edmonton, Alberta, Canada T6G 2G1
}

\begin{abstract}
We study the repeated folding of a two-parameter family of quadrilaterals about their successively transformed diagonals by examining the evolution of the diagonal lengths. Successively mapped pairs of squared lengths lie on an elliptic curve on which folding acts as translation under the group law. We prove the rotation number attains all possible values and any value determines a unique curve in parameter space. For rational parameters we give an algorithm to determine if the folding map is periodic. This gives a partial explanation for the diversity and intricacy of the curves traced out by the paths of the vertices of the transformed quadrilaterals.
\end{abstract}

\section{Introduction}

Thefolding of a planar polygon as a visual phenomenon and as a formally defined dynamical system on $\mathbb{C}^{n}$ (vertex space) was introduced in [CR]. Take a polygon and fold it back into the same plane about one of its diagonals. If you are lucky, you can continue this activity, producing a succession of folded polygons (following some selection scheme or other for the diagonals). The scheme we select is recursive in nature, and with few exceptions it results in endless folding. The path of the sides of the successively transformed polygons resembles the motion one associates with a screensaver program, although we are more interested here in the motion of the vertices. We call the union of the vertices generated in such a fashion a polypath.

It is not difficult to select a quadrilateral, say, and with the simple cyclic rule of folding described below produce a polypath which appears to be dense on an intricate planar curve, replete with multiple components, self-intersections, loops, variable curvature. Such a curve tends to have what might be called local structure: collections of subarcs with complicated geometry (though not on all scales). Furthermore, as one varies the initial quadrilateral (the one we start out folding with) an astonishing diversity of curves is encountered. For a small sample, see Fig. 1.

\footnotetext{
* This research was supported by a grant from NSERC.
} 

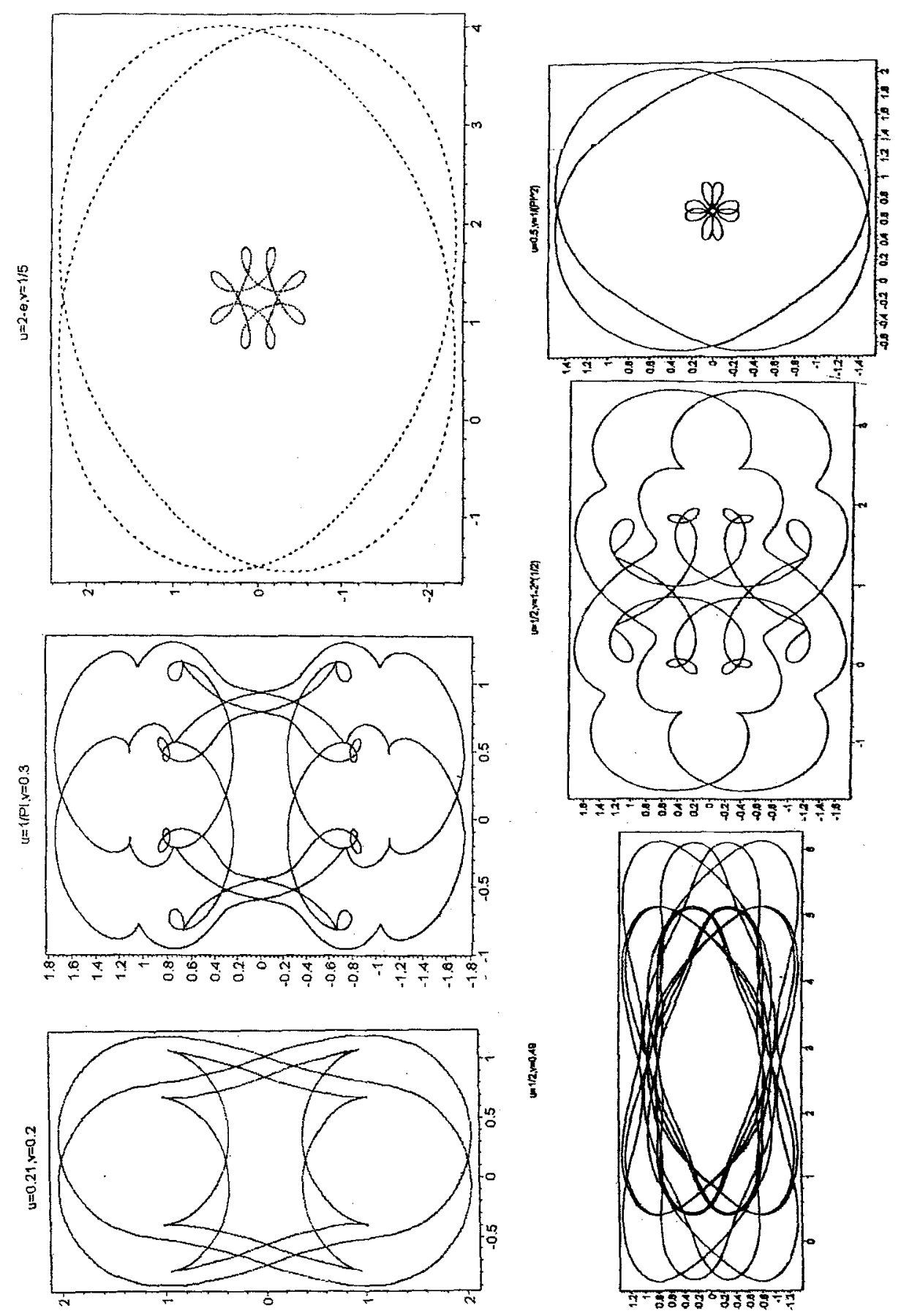

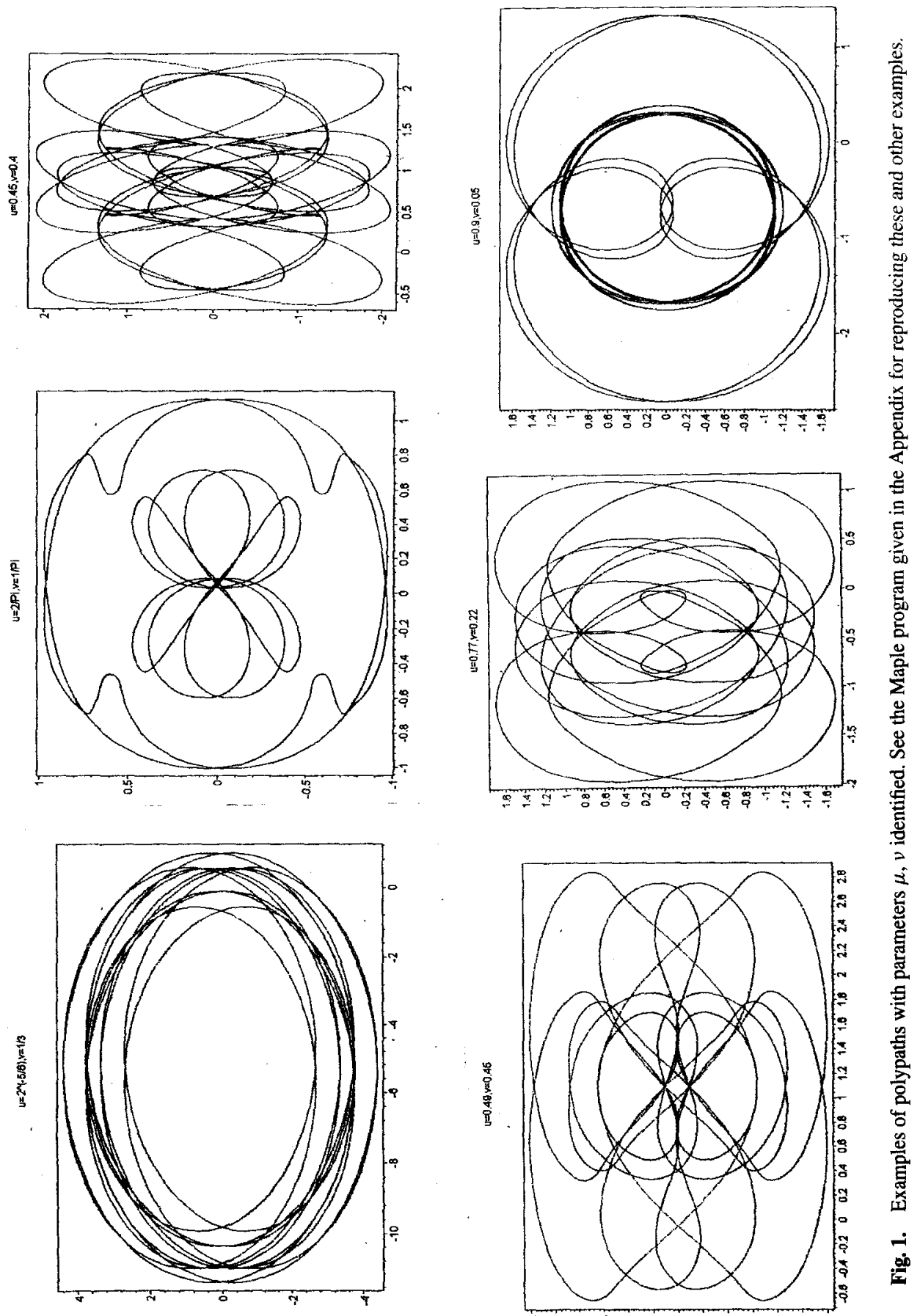
Polypaths are associated with a simple, geometrically conceived dynamical system with behavior that is complex but easily visualized. This is their charm and this is why we study them.

Putting aside the explicit rule of folding for a moment, in this paper (as in [CR]) we restrict the initial set of polygons to a coordinatized class of isosceles trapezoids with labeled vertices satisfying an isoperimetric constraint. The subsequently folded images are in general quadrilaterals whose sides retain the same length, but whose diagonal lengths vary (as do their position and orientation). Thus, any two such folded quadrilaterals with equal diagonals are transformable, the one to the other, by a motion acting rigidly on their sides.

The crucial property of the resulting system is that the squared diagonal lengths lie on the closed component of a real elliptic curve. The dynamical process relating subsequent pairs is translation under the group law on the elliptic curve. Thus essential properties of folding can be modeled by an algebraic dynamical system. The translational constant varies with the same two parameters which characterize the initial set of trapezoids. Our polypaths thus provide a novel application of the classical theory of real elliptic curves, perhaps akin to some of the mechanical examples in [MM]. Our main result is that the rotation number of the conjugate family of translations of the circle varies monotonically along certain curves in parameter space (so there are no constant portions, no "devil's staircases" associated with rational rotation number). This result provides a reason forand strongly complements-the numerical evidence of the complexity and diversity of polypaths.

In Section 1 we review the necessary background (with a number of amendments). We derive a Weierstrass form of the elliptic curve which has a polynomial discriminantthis eases the subsequent calculations of rotation number (the discriminant in [CR] is rational). We also correct a computational error in the basic demonstration that the diagonal dynamics are equivalent to translation on an elliptic curve. Section 2 reduces the problem to the study of a two-parameter family of translations on the circle. (No doubt there is a useful general bifurcation theory for such systems, but for our application there is no avoiding the fact that we must deal with the classical analysis of elliptic functions.) We show that parameter space may be reduced to a subset $\mathcal{T}$, and using the Weierstrass $\wp$ function find the period rectangle which enables us to derive a formula for the parameterdependent rotation number $\rho(\mu, \nu)$. We prove that $\rho$ is a smooth function on $\mathcal{T}$, and this result is used to show that the dynamics of quadrilateral folding are in part describable by the appropriately defined folding of a "degenerate quadrilateral"-in fact a triangle. The main result is that for any value $\rho_{0} \in(0,1)$, there is a curve $\mu=f(v)$ defined on the basic parameter space $\mathcal{T}$ such that $\rho(\mu, v)=\rho_{0}$. Thus the rotation number takes on all possible values and has no constant portions, which provides a reasonable explanation for the complexity of polypaths. Section 3 studies the case of rational orbits on the closed component $\Sigma$ of the elliptic curve, providing with the help of the Lutz-Nagel and Mazur theorems a useful condition under which the translation has infinite period. As a corollary, for $(\mu, v) \in \mathcal{T} \cap \mathbb{Q}^{2}$, either the folding iterated $n$ times acts as a rigid motion for some $n$ with $1 \leq n \leq 48$ or else the initial quadrilateral never recurs. In Section 4 we consider the simple rational map of the plane $(x, y) \mapsto(y,(y+\alpha) / x)$ and show it is conjugate to double iteration of the elliptic curve translation arising in the polypath 
setting. Section 5 concludes with a number of unsolved problems and some suggestions for further research.

\section{The Folding of Quadrilaterals and Translation on an Elliptic Curve}

A directed quadrilateral, labeled $P Q R S$, is an ordered 4-tuple of points (vertices) in the plane, where the order indicates a clockwise orientation of the points. We may reflect the vertex $P$ over the diagonal determined by its adjacent vertices provided $Q \neq S$, producing a new directed quadrilateral $Q R S P^{\prime}$. Cyclic folding is the map of quadrilaterals

$$
P Q R S \rightarrow Q R S P^{\prime}
$$

where $P^{\prime}$ is the reflected image of $P$ about diagonal $Q S$. If the initial quadrilateral has no adjacent sides of equal length, cyclic folding can be continued indefinitely; otherwise we call the quadrilateral a dart. (Eliminating all quadrilaterals with two adjacent sides of equal length is somewhat more restrictive than necessary, but it is expedient and quite consistent with our further reductions in generality.) The set of quadrilaterals which are not darts is easily seen to be an open subset of $\left(\mathbb{R}^{2}\right)^{4}=\mathbb{C}^{4}$. Let $\left.P\right|_{Q} ^{S}$ denote the reflection of $P$ in the line $Q S$. Identifying $\mathbb{R}^{2}$ with $\mathbb{C}$, one may verify the correspondence

$$
\left.P\right|_{Q} ^{S}=\frac{\overline{P-\bar{Q}}(S-Q)}{\overline{S-Q}}+Q
$$

(where the bar indicates complex conjugation).

This defines a map $\pi$ on the open set of nondarts,

$$
\pi(P Q R S)=\left.Q R S P\right|_{Q} ^{S}
$$

It is easily verified that $\pi$ is a real analytic diffeomorphism with inverse $r \circ \pi \circ r$ where $r$ is the involution $r(P Q R S)=S R Q P . \pi$ has Jacobian determinant equal to 1 and so preserves Lebesgue measure in $\mathbb{C}^{4}$. We may now speak of (forward and backward) orbits of (nondart) directed quadrilaterals under $\pi$.

The map $\pi$ preserves the "side lengths" of a quadrilateral. More precisely, if we define a map to 4-tuples of positive reals by $D_{i}=\left|P_{i+1}-P_{i}\right|, i=0,1,2,3$, with mod 4 addition of indices and with $P_{0}=P, P_{1}=Q, P_{2}=R, P_{3}=S$, then we see that $\pi$ acts as a cyclic permutation on these 4-tuples.

Speaking loosely, $\pi$ does two things to a quadrilateral. First, it changes the position of one of the vertices relative to the other three-that is, it changes the shape. Second, it changes the position of the quadrilateral as a whole, say as measured by the coordinates of the centroid relative to some coordinate system. Although there are some interesting questions associated with the latter (for example, can you tell by looking at an initial quadrilateral if its $\pi$-orbit will remain bounded?), we have chosen to focus on the former. From this point of view we have a four-parameter dynamical system, indexed by the four side lengths $D_{i}$ mentioned above. Since we are interested only in the similarity class of 

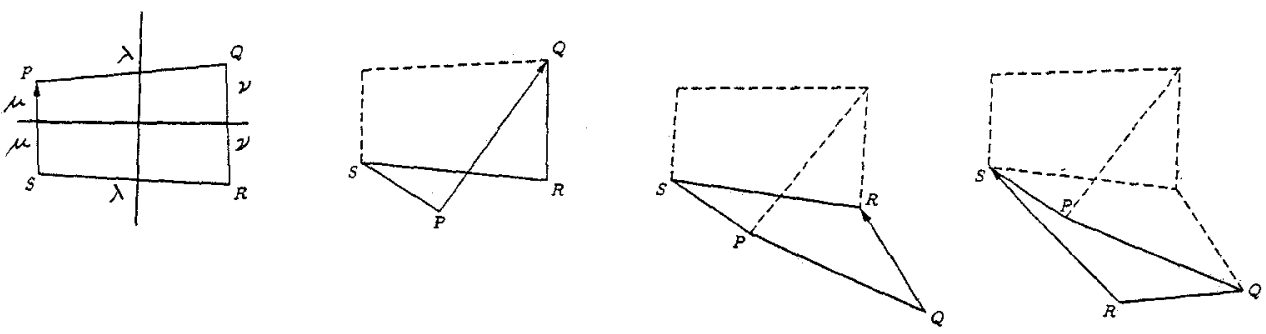

Fig. 2. The cyclic folding rule.

a given quadrilateral, overall size is irrelevant and so we impose the constraint that the perimeter equal 4 (that of a unit square). This reduces us to a three-parameter family of quadrilaterals. We will find ourselves doing calculus with fairly complicated functions of these parameters (e.g., classical elliptic functions), and this analysis is simplified by reduction to two parameters. We do this by reducing the class of initial quadrilaterals that we consider, and our experience has shown that the dynamics are still rich enough to make this reduction in generality worthwhile.

That said, we now spell out our choice of initial quadrilaterals: we study initial isosceles trapezoids of perimeter 4 centered on the origin of the plane (which we identify with $\mathbb{C}$ or $\mathbb{R}^{2}$ as is convenient). While the trapezoidal nature of these figures is not preserved by $\pi$, the property that a pair of opposite sides have the same length is (as is the isoperimetric restriction). The side lengths are labeled $2 \mu, \lambda, 2 \nu, \lambda$ (see Fig. 2). (The factors of 2 are inserted to simplify the coordinates of the vertices given below.) Our perimeter constraint reads $\mu+v+\lambda=2$, and we consistently write $2-\mu-v$ in place of $\lambda$.

Clearly we need $(\mu, \nu) \in[0,1] \times[0,1]$. If $\mu$ or $\nu$ is 0 , we have an isosceles triangle; if $\mu$ or $\nu$ is 1 , we have four collinear points. Also, if $\mu=v=\frac{1}{2}$ we have a square. For our purposes then, we restrict $(\mu, \nu)$ to the open unit square, omitting the point $\left(\frac{1}{2}, \frac{1}{2}\right)$. Call this set $\mathcal{U}$. For $(\mu, v) \in \mathcal{U}$ let

$$
\begin{aligned}
& v_{0}=-\sqrt{1+\mu \nu-\mu-v}+i \mu, \\
& v_{1}=\sqrt{1+\mu \nu-\mu-v}+i v, \\
& v_{2}=\bar{v}_{1} \\
& v_{3}=\bar{v}_{0} .
\end{aligned}
$$

Then $\left(v_{0}, v_{1}, v_{2}, v_{3}\right)$ is an initial directed quadrilateral. The folding map $\pi$ then has the forward orbit given by

$$
\pi\left(v_{i}, v_{i+1}, v_{i+2}, v_{i+3}\right)=\left(v_{i+1}, v_{i+2}, v_{i+3}, v_{i+4}\right),
$$

$i=0,1,2, \ldots$, where $v_{i+4}=\overline{\left(v_{i}-v_{i+1}\right)}\left(v_{i+3}-v_{i+1}\right) / \overline{\left(v_{i+3}-v_{i+1}\right)}+v_{i+1}$, and the inverse (backward) orbit is generated by $r \circ \pi \circ r$ (where $r$ is the involution defined previously).

Since we are only interested in the relative positions of the vertices of our quadrilateral, and since $\pi$ preserves side lengths, we can study the folding dynamics by studying the 
diagonal dynamics. That is, given vertices $P, Q, R, S$ in the plane, we define $x=$ $|P-R|^{2}, y=|Q-S|^{2}$ (the squared diagonal lengths), and we ask how $x$ and $y$ evolve under $\pi$. This passage to diagonal dynamics is crucial to all that follows. The reason for this is that $x, y$ and the side lengths $D_{i}, i=0,1,2,3$, satisfy the identity

$$
\begin{aligned}
& x y^{2}+x^{2} y-\left(D_{0}^{2}+D_{1}^{2}+D_{2}^{2}+D_{3}^{2}\right) x y+\left(D_{0}^{2}-D_{3}^{2}\right)\left(D_{1}^{2}-D_{2}^{2}\right) x \\
& \quad+\left(D_{0}^{2}-D_{1}^{2}\right)\left(D_{3}^{2}-D_{2}^{2}\right) y+\left(D_{1}^{2} D_{3}^{2}-D_{0}^{2} D_{2}^{2}\right)\left(D_{1}^{2}+D_{3}^{2}-D_{0}^{2}-D_{2}^{2}\right)=0 .
\end{aligned}
$$

(This was originally obtained by considering the formula for the volume of a parallelepiped spanned by $Q-P, R-P, S-P$, which is 0 . See [CR].) This is easily verified by expressing all defined quantities in terms of $P, Q, R, S$ and then expanding the left-hand side. Notice also that if the coefficients of this polynomial in $x$ and $y$ are determined by some initial quadrilateral, then under folding $\left.P Q R S \rightarrow Q R S P\right|_{Q} ^{S}$ and then relabeling $Q \rightarrow P, R \rightarrow Q, S \rightarrow R,\left.P\right|_{Q} ^{S} \rightarrow S$ we see that $x$ and $y$ are interchanged, and the permutation $D_{0} \rightarrow D_{3}, D_{1} \rightarrow D_{0}, D_{2} \rightarrow D_{1}, D_{3} \rightarrow D_{2}$ occurs. Then the reader can check the invariance property that the overall effect of these permutations on the above polynomial is null--in other words, the same coefficients are valid under any number of iterations of $\pi$.

For our class of initial trapezoids, we have $x=\left|v_{0}-v_{2}\right|^{2}, y=\left|v_{1}-v_{3}\right|^{2}$. The relation above takes the form

$$
x^{2} y+x y^{2}-Q x y-L(x+y)+C=0
$$

where the coefficients are polynomials in $\mu, \nu$ :

$$
\begin{aligned}
& Q=4\left(\mu^{2}+v^{2}\right)+2(2-\mu-v)^{2}, \\
& L=\left(4 \mu^{2}-(2-\mu-v)^{2}\right)\left(4 v^{2}-(2-\mu-v)^{2}\right), \\
& C=\left(16 \mu^{2} \nu^{2}-(2-\mu-v)^{4}\right)\left(4\left(\mu^{2}+v^{2}\right)-2(2-\mu-v)^{2}\right) .
\end{aligned}
$$

The resulting curve, denoted $X_{\mu v}$, is symmetric with respect to the line $x=y$ and is asymptotic to the lines $x=0, y=0$, and $x+y=Q$. The triangular region enclosed by the asymptotes contains a closed component of $X_{\mu \nu}$ which we conveniently call the shoe, denoted $\Sigma$.

The shoe intersects the line $x=y$ twice, once at the point $\left(x_{0}, y_{0}\right)$ corresponding to the initial trapezoid. When $X_{\mu \nu}$ is projectivized, it has three points at infinity: $[1,0,0]$, $[0,1,0],[1,-1,0]$. The last point is an inflection point. The curve meets the axes at the points $(0, C / L)$ and $(C / L, 0)$. When $L=0$ this intersection is at infinity. See Fig. 3(a).

If $\mu-v \neq 0$ and $\mu+v \neq 1$, then $X_{\mu \nu}$ is actually a real elliptic curve. More precisely, the projective transformation

$$
\varphi:\left\{\begin{array}{l}
X \rightarrow\left(\frac{Q^{2}-4 L}{24}\right) X+\left(\frac{Q^{2}-4 L}{24}\right) Y+\left(\frac{12 C-8 L Q-Q^{3}}{24}\right) Z, \\
Y \rightarrow\left(\frac{C-L Q}{2}\right) X-\left(\frac{C-L Q}{2}\right) Y, \\
Z \rightarrow \frac{1}{2} X+\frac{1}{2} Y-\frac{Q}{2} Z
\end{array}\right.
$$




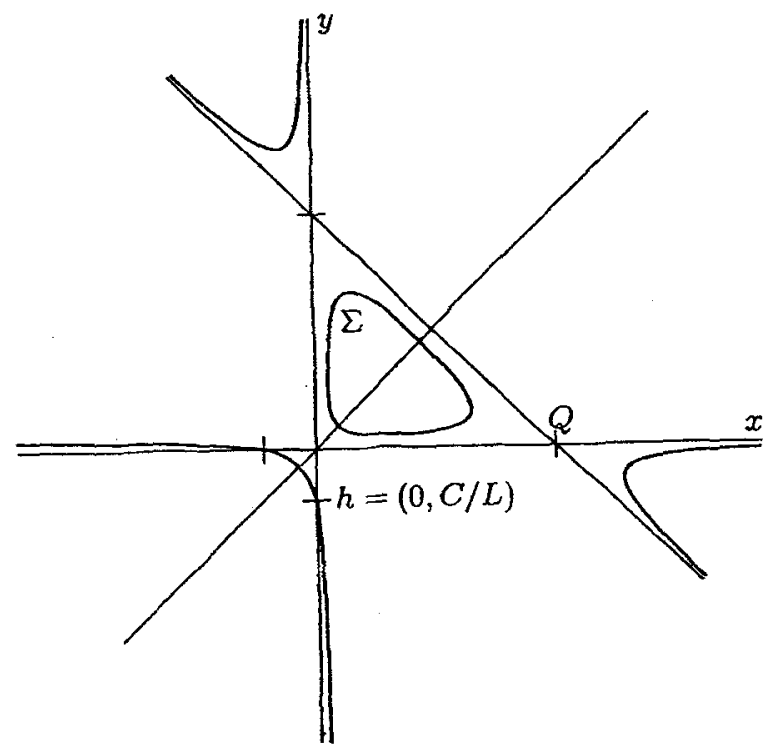

(a)

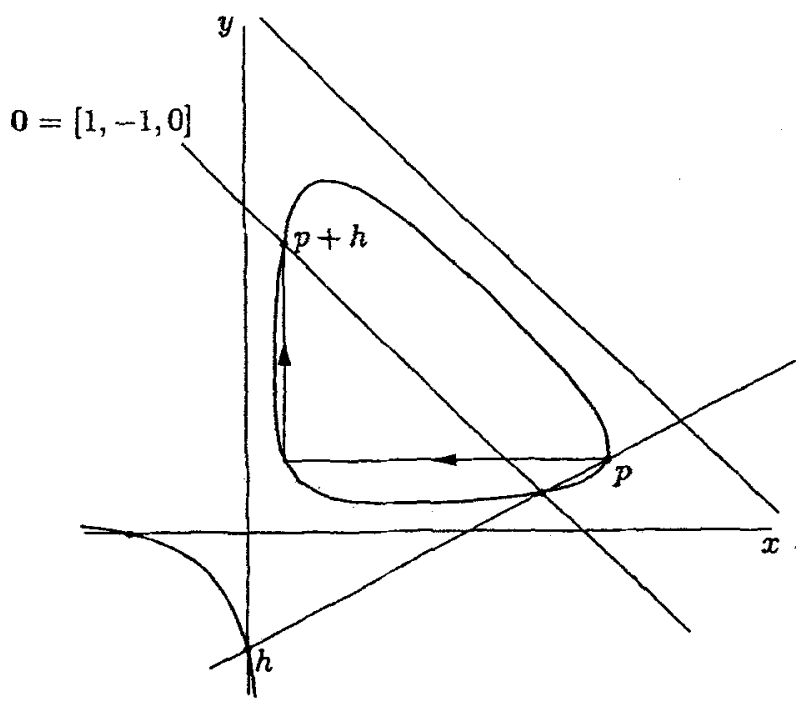

(b)

Fig. 3. (a) The elliptic curve $X_{\mu \nu}$. (b) Translation on the shoe $\Sigma$ is equivalent to unconventional billiards.

transforms the projective completion of $X_{\mu \nu}$ to the Weierstrass normal form $W_{\mu \nu}$,

$$
Y^{2} Z=4 X^{3}-g_{2}(\mu, \nu) X Z^{2}-g_{3}(\mu, \nu) Z^{3},
$$

where

$$
\begin{aligned}
& g_{2}=\frac{1}{12} Q^{4}+\frac{4}{3} Q^{2} L+\frac{4}{3} L^{2}-2 Q C \\
& g_{3}=-\frac{1}{216} Q^{6}-\frac{1}{9} Q^{4} L+\frac{1}{6} Q^{3} C-\frac{5}{9} L^{2} Q^{2}+\frac{8}{27} L^{3}+\frac{4}{3} C L Q-C^{2}
\end{aligned}
$$


provided $C-L Q \neq 0$. The transformation (which is different from the one of [CR]) has the advantage of yielding a polynomial discriminant:

$$
\begin{aligned}
\Delta & =\Delta(\mu, v) \\
& =268435456 \mu^{2} v^{2}(1-\mu)^{2}(1-v)^{2}(1-\mu-v)^{2}(\mu+v)^{4}(\mu-v)^{4}(2-\mu-v)^{4} .
\end{aligned}
$$

The polynomial form of $\Delta$ greatly eases the subsequent analysis. The locus of $C-L Q=$ 0 is the union of the lines $\mu+v=0, \mu-\nu=0$, and $\mu+\nu=2$. If we also avoid the line $\mu+\nu=1$, we see that $\Delta>0$.

As a consequence, if we remove the lines $\mu-v=0$ and $\mu+v=1$ from the open square $\mathcal{U}$ we see that $X_{\mu \nu}$ is a real elliptic curve, and further that the right-hand side of the Weierstrass form has three real roots [K] (hence this form has two components). The projectivity sends the shoe $\Sigma$ to the bounded component (a simple closed curve) and the point $h=(0, C / L)$ to the point $H=\left(\left(Q^{2}+8 L\right) / 12,-C\right)$ on the unbounded component of the Weierstrass form of the curve.

What are the dynamics on the shoe $\Sigma$ corresponding to iteration of the polypath mapping $\pi$ ? Choosing a parameter pair $(\mu, v)$ amounts to choosing an initial trapezoid, which corresponds to a point on $\Sigma$ (the ordered pair of squared diagonals $(x, y)$ ). There are two ways of looking at the subsequent orbit. Folding fixes one of the diagonals. Thus, the corresponding action on $X_{\mu \nu}$ is to move along a horizontal or vertical line to another point on $X_{\mu \nu}$. Under continual iteration of $\pi$, we alternate between horizontal and vertical motion. A line through a point $\left(x_{0}, y_{0}\right)$ on the shoe drawn parallel to one of the axes will intersect $X_{\mu \nu}$ at one of the points of infinity, $[1,0,0]$ or $[0,1,0]$ in this case, and at one other point, by Bezout's theorem. Since the shoe is a closed curve, the third point will either be a new point on the shoe or, in the case that the line is tangent to the shoe, it will be $\left(x_{0}, y_{0}\right)$ itself. Thus folding generates a billiard-like path on the shoe, rebounding alternately in horizontal and vertical directions. (In the case of tangency, the particle "spins," and then moves on.) See Fig. 3(b).

The second way of regarding shoe dynamics is essentially algebraic. $X_{\mu \nu}$ is a real elliptic curve and so has a natural group structure-addition on an elliptic curve-defined by chord-and-tangent constructions. Briefly, given $A=\left(x_{1}, y_{1}\right), B=\left(x_{2}, y_{2}\right)$ on the Weierstrass curve $y^{2}=4 x^{3}-g_{2} x-g_{3}$, find the third intersection of $A B$ with the curve (if $A=B$, use the tangent line at $A$ ), then reflect in the $x$-axis to obtain $A+B$; a real elliptic curve which is the image of such a Weierstrass curve under a projective transformation has an addition induced by this transformation (see [ST]). The group is defined through selection of its zero element: we select the point at infinity $O=[1,-1,0]$. (Under the projectivity to the Weierstrass form, $O$ maps to the unique point at infinity $[0,1,0]$.) $O$ is an inflection point, so three points on $X_{\mu \nu}$ are collinear if and only if they add to $O$.

We shall see that two applications of folding corresponds to translation of the points on $\Sigma$ by the point $h=(0, C / L)$ (which lies on the unbounded component). See Fig. 3 . Let $p \in \Sigma$, let $p^{\prime}$ be the horizontal billiard image of $p$, and let $p^{\prime \prime}$ be the vertical billiard image of $p^{\prime}$. From above, we have the relations (addition on the cubic)

$$
\begin{aligned}
& O=p+p^{\prime}+[1,0,0], \\
& O=p^{\prime}+p^{\prime \prime}+[0,1,0], \\
& O=[1,0,0]+[0,1,0] .
\end{aligned}
$$


Combining these we obtain

$$
p^{\prime \prime}=p+2[1,0,0]
$$

This calculation corrects an error in calculation in [CR]. Recall that $X_{\mu \nu}$ intersects the $x$-axis twice at $[1,0,0]$ and once at $(C / L, 0)$ (projectively, $[C, 0, L])$. Thus the doubling $2[1,0,0]$ is the reflection of $(C / L, 0)$ in the line $y=x$, which of course is $h=(0, C / L)$. Thus from the viewpoint of diagonal dynamics, applying $\pi^{2}$ (folding a quadrilateral twice in succession) is equivalent to the translation

$$
\tau: p \mapsto p+h
$$

on the shoe $\Sigma$.

The real elliptic curve $X_{\mu \nu}$ is analytically isomorphic (via the Weierstrass $\wp$-function) to $\mathbb{R} / 2 \pi \mathbb{Z} \times \mathbb{Z} / 2 \mathbb{Z}$. $\Sigma$ is isomorphic to $\mathbb{R} / 2 \pi \mathbb{Z}$, as is the unbounded component containing $h$. Hence $\tau$ is actually translation on a circle by some angle $2 \pi \rho$, with $\rho=\rho(\mu, v) \in$ $[0,1)$, and so we end up with a two-parameter family of translations on the circle $S^{1}$. Consequently, the diagnonal dynamics are completely determined by the values assumed by the rotation number $\rho$. See [D] or [M]. If $\rho$ is rational, $\tau$ has finite period on the shoe $\Sigma$. This implies that the folding eventually produces a quadrilateral congruent to the initial trapezoid, though possibly in a different orientation and a different location. If $\rho$ is irrational, the orbit of $\tau$ is dense on $\Sigma$. We study the properties of $\rho$ in more detail in the next section.

\section{The Rotation Number}

The main result of this section is that given any $\rho_{0} \in(0,1)$, there is a unique continuous curve along which $\rho(\mu, v)=\rho_{0}$. This demonstrates that the dynamics are indeed "sensitive to initial conditions" (i.e., there are no stable regions), and yet for a given $\mu$ or $v$ we can always find a value of the other parameter with the desired rotation number.

As a consequence, for any properly defined "transversal curve" $\mathbf{C}$ in parameter space and any value $\rho_{0} \in(0,1)$, there is a point on $\mathbf{C}$ such that the corresponding member of the family $(\Sigma, \tau)$ is conjugate to translation on the circle by the angle $2 \pi \rho_{0}$.

We note first that replacing $(\mu, v)$ by $(\nu, \mu)$ or $(1-\mu, 1-\nu)$ leaves $Q, L, C$ unchanged and so leaves $X_{\mu \nu}$ and $h$ unchanged. For this reason it suffices to restrict our study of $\rho(\mu, \nu)$ to the open triangle $\mathcal{T}$ defined by $\nu>0, v<\mu, \nu<1-\mu$. (See Fig. 4.)

Next we note that we may deal with the Weierestrass form $W_{\mu \nu}$ of $X_{\mu \nu}$ given previously. Indeed $\operatorname{det}(\varphi)=((C-L Q) / 2)^{2} \neq 0$ and so $\varphi$ is a projective transformation for $(\mu, \nu) \in \mathcal{T}$. Moreover, $\varphi(O)=\varphi([1,-1,0])=[0,1,0]$. It then follows (e.g., see [S2]) that $X_{\mu \nu}$-with the group structure we have imposed on it-is isomorphic to $W_{\mu \nu}$ with the standard group structure (i.e., $O=[0,1,0]$ ).

Before proceeding to the general case we consider two specific situations mentioned in [CR].

Example 1. The condition $\rho=\frac{1}{2}$ is equivalent to $2 h=O$, which is equivalent to $2 H=O$ (recall that $H=\varphi(h)=\left(\left(Q^{2}+8 L\right) / 12,-C\right)$ ). For the Weierstrass form 


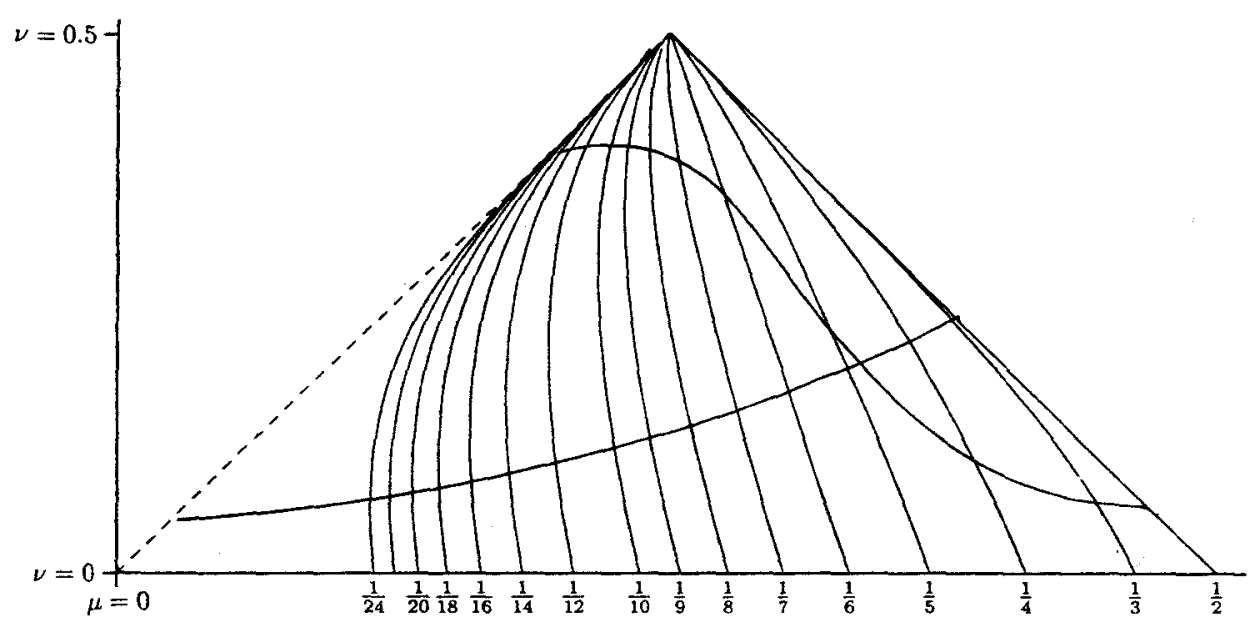

Fig. 4. Curves in the parameter region $\mathcal{T}$ associated with various rational rotation numbers $\rho$, decorated with two transversals (see Corollary 2).

$W_{\mu \nu}$, a point has order 2 if and only if it lies on the $x$-axis. Thus we see that $\rho=\frac{1}{2}$ precisely when $C=0$. This expression factors to give

(i) $\mu^{2}+v^{2}-2 \mu v-4 \mu-4 v+4=0$

or

(ii) $\mu^{2}+v^{2}-2 \mu v+4 \mu+4 v-4=0$.

In the parameter region $\mathcal{T}$ the relevant curve is given by a piece of (ii):

$$
v=\mu+2^{3 / 2} \sqrt{1-\mu}-2 \text {. }
$$

So in this case we have an explicit algebraic curve in $\mathcal{T}$ defined by $\rho=\frac{1}{2}$.

The corresponding folding behavior is that $\pi^{4}$ acts as a rotation by $180^{\circ}$, so $\pi^{8}$ is the identity transformation. Interestingly, although the dynamics on the shoe $\Sigma$ are the same on the curve (i), in this case $\pi^{8}$ acts as a translation, so the corresponding polypath is unbounded. This highlights the fact that the diagonal dynamics are only part of the story.

Example 2. As a second special case, consider the situation when $L=0$. This factors to give (i) $\nu=2-3 \mu$, or (ii) $\nu=\frac{2}{3}-1 / 3 \mu$.

Only (i) intersects $\mathcal{T}$. Along this line, $h$ is a point at infinity $(h=[0, C, L]=$ $[0, C, 0]=[0,1,0])$. From the previous section, $h=2[1,0,0]$. Thus we have $2 h=$ $2[0,1,0]=[1,0,0]=-2[1,0,0]=-h$ (since when $L=0, X_{\mu \nu}$ intersects each axis triply at infinity). We conclude that $3 h=0$, i.e., $h$ has order 3 . Thus $\nu=2-3 \mu$ defines either $\rho=\frac{1}{3}$ or $\frac{2}{3}$ (we will show it is the former). In this case, $\pi^{12}$ acts as a translation, and the polypath is unbounded. 
The general study of the rotation number requires us to make use of the analytic isomorphism-via the Weierstrass $\wp$-function-between a complex elliptic curve and its period parallelogram in $\mathbb{C}$ (i.e., torus) (see [K] and [ST]).

For $(\mu, v) \in \mathcal{T}$, the discriminant $\Delta>0$ (unless otherwise stated, from now on we are dealing with the Weierstrass form $W_{\mu \nu}$ ). Thus $W_{\mu \nu}$ has three distinct real roots, $e_{1}<e_{3}<e_{2}$, and the period parallelogram can be taken to be the rectangle generated by

$$
\omega_{1}=2 i \int_{-\infty}^{e_{1}} \frac{d t}{\sqrt{g_{3}+g_{2} t-4 t^{3}}}
$$

and

$$
\omega_{2}=2 \int_{e_{2}}^{\infty} \frac{d t}{\sqrt{4 t^{3}-g_{2} t-g_{3}}},
$$

where $g_{2}=g_{2}(\mu, v)$ and $g_{3}=g_{3}(\mu, \nu)$ are defined above in terms of $Q, L, C$. Explicitly we have

$$
\begin{aligned}
g_{2}= & \left(v^{2}-v+1\right) \mu^{6}+\left(4 v^{3}-9 v^{2}+3 v-2\right) \mu^{5}+\left(6 v^{4}-22 v^{3}+23 v^{2}-2 v+1\right) \mu^{4} \\
& +2 v^{2}\left(2 v^{3}-11 v^{2}+21 v-14\right) \mu^{3}+v^{2}\left(v^{4}-9 v^{3}+23 v^{2}-28 v+14\right) \mu^{2} \\
& -v^{4}\left(v^{2}-3 v+2\right) \mu+v^{4}\left(v^{2}-2 v+1\right), \\
g_{3}= & -\frac{16384}{27}\left((2 v-1) \mu^{3}+\left(4 v^{2}-7 v+1\right) \mu^{2}+v\left(2 v^{2}-7 v+6\right) \mu-v^{2}(v-1)\right) \\
& \times\left((v+1) \mu^{3}+\left(2 v^{2}-5 v-1\right) \mu^{2}+v\left(v^{2}-5 v+6\right) \mu+v^{2}(v-1)\right) \\
& \times\left((v-2) \mu^{3}+2\left(v^{2}-v+1\right) \mu^{2}+v^{2}(v-2) \mu-2 v^{2}(v-1)\right) .
\end{aligned}
$$

The Weierstrass correspondence sends the base of this period rectangle to the unbounded component of the real elliptic curve $W_{\mu \nu}$, which is the component containing the translational constant $H$. The bisector of the rectangle parallel to the real axis, $\operatorname{Im} z=\left|\omega_{1}\right| / 2$, is sent to the bounded (closed) component. Since the opposite sides of the rectangle are identified, these "line segments" are actually circles. The translation $\tau: p \mapsto p+H$ on $W_{\mu \nu}$ is conjugate to the translation of the inverse image of $p$ on its circle by the angle subtended by the inverse image of $H$ on its circle. The ratio of this angle to $2 \pi$ is equal to the ratio of $\wp^{-1}(H)$ (modulo the lattice generated by $\left(\omega_{1}, \omega_{2}\right)$ ) to $\omega_{2}$. Since

$$
\wp^{-1}(H)=\int_{H_{x}}^{\infty} \frac{d t}{\sqrt{4 t^{3}-g_{2} t-g_{3}}},
$$

where $H=\left(H_{x}, H_{y}\right)$, we obtain the the following proposition.

Proposition 1. Let $(\mu, v) \in \mathcal{T}$ and $g_{2}, g_{3}, e_{2}, H_{x}$ be as above. Then

$$
\rho(\mu, v)=\frac{\int_{H_{x}}^{\infty}\left(d t / \sqrt{4 t^{3}-g_{2} t-g_{3}}\right)}{2 \int_{e_{2}}^{\infty}\left(d t / \sqrt{4 t^{3}-g_{2} t-g_{3}}\right)},
$$

where the integrals are improper Riemann integrals. 
The study of $\rho(\mu, \nu)$ on $\mathcal{T}$ requires us to examine the parameter dependence of the roots of $4 t^{3}-g_{2} t-g_{3}$. It turns out that they are polynomials in $\mu$ and $\nu$. Indeed, some tedious algebra gives

$$
\begin{aligned}
& e_{1}=\frac{16}{3}\left[(1-2 v) \mu^{3}-\left(4 v^{2}-7 v+1\right) \mu^{2}-v\left(2 v^{2}-7 v+6\right) \mu+v^{2}(v-1)\right] \\
& e_{2}=\frac{16}{3}\left[(v-2) \mu^{3}+2\left(v^{2}-v+1\right) \mu^{2}+v^{2}(v-2) \mu-2 v^{2}(v-1)\right] \\
& e_{3}=\frac{16}{3}\left[(v+1) \mu^{3}+\left(2 v^{2}-5 v+1\right) \mu^{2}+v\left(v^{2}-5 v+6\right) \mu+v^{2}(v-1)\right] .
\end{aligned}
$$

If we put

and define $\varphi$ by

$$
\lambda=\frac{e_{3}-e_{1}}{e_{2}-e_{1}}=\frac{\mu \nu(2-\mu-\nu)^{2}}{(1-\mu)(1-\nu)(\mu+\nu)^{2}}
$$

$$
\cos \varphi=-\left(\frac{v^{2}+\mu^{2}-2 \mu \nu+4 \mu+4 v-4}{v^{2}+\mu^{2}+6 \mu \nu-4 \mu-4 v+4}\right)
$$

so that $\cos ^{2} \varphi=\left(H_{x}-e_{2}\right) /\left(H_{x}-e_{1}\right)$, then the canonical transformations for elliptic integrals of the first kind (see p. 597 of [AS], although we retain the notation of Koblitz $[\mathrm{K}])$ gives us the following alternative form:

$$
\rho(\mu, \nu)=\frac{\int_{0}^{\varphi}\left(d \theta / \sqrt{1-\lambda \sin ^{2} \theta}\right)}{2 \int_{0}^{\pi / 2}\left(d \theta / \sqrt{1-\lambda \sin ^{2} \theta}\right)}=\frac{F(\varphi ; \lambda)}{2 K(\lambda)},
$$

where $F(\varphi ; \lambda)$ and $K(\varphi)$ are incomplete and complete elliptic integrals of the first kind, respectively.

In order to understand $\rho(\mu, v)$ we need to understand $\varphi=\varphi(\mu, v)$ and $\lambda=\lambda(\mu, v)$ on $\mathcal{T}$. (Note that both functions are defined on $\mathcal{T}$.)

Simple calculations give us the symmetrically related partial derivatives (note $\partial f / \partial \mu(\mu, \nu)=$ $\partial f / \partial v(\nu, \mu))$,

$$
\begin{aligned}
& \frac{\partial \lambda}{\partial \mu}=\frac{\nu(\nu-\mu)(2-\mu-v)(2-3 \mu-v)}{(1-\mu)^{2}(1-v)(\mu+v)} \\
& \frac{\partial \lambda}{\partial \nu}=\frac{\mu(\mu-v)(2-\mu-v)(2-3 v-\mu)}{(1-v)^{2}(1-\mu)(\mu+v)} .
\end{aligned}
$$

The partials exist and are continous on (say) $S=(0,1) \times\left(0, \frac{1}{2}\right)$, and $\mathcal{T} \subset S$. This implies that $\lambda(\mu, v) \in C^{1}(S)$. Moreover, we see that $\lambda=1$ when $v=\mu, \nu=1-\mu$ $(\mu \in(0,1))$, and that $\lambda \in(0,1)$ when $(\mu, \nu) \in \mathcal{T}$. A similar calculation shows that

$$
\begin{aligned}
& \frac{\partial \varphi}{\partial \mu}=\frac{2(2-\mu+v) \sqrt{1-\nu}}{\left(\mu^{2}+v^{2}+6 \mu \nu-4 \mu-4 v+4\right) \sqrt{1-\mu}}, \\
& \frac{\partial \varphi}{\partial \nu}=\frac{2(2-v+\mu) \sqrt{1-\mu}}{\left(\mu^{2}+v^{2}+6 \mu \nu-4 \mu-4 v+4\right) \sqrt{1-\nu}}
\end{aligned}
$$

(also symmetrically related) and hence $\varphi(\mu, \nu) \in C^{1}(S)$. 
For a fixed $\nu \in\left(0, \frac{1}{2}\right), \varphi$ increases as we move from left to right across $\mathcal{T}$. Also, $\varphi=\pi / 2$ precisely when $\mu^{2}+v^{2}-2 \mu v+4 \mu+4 v-4=0$, and recalling that this is also the relation defining $2 H=0$, the value $\varphi=\pi / 2$ clearly gives $\rho(\mu, \nu)=\frac{1}{2}$, in accord with our earlier ad hoc result, Example 1.

Finally here, to prove $\rho$ is $C^{1}$ on $\mathcal{T}$, we make use of three classical formulas [SO], namely

$$
\begin{aligned}
& \frac{\partial F}{\partial \varphi}=\frac{1}{\sqrt{1-\lambda \sin ^{2} \varphi}}, \\
& \frac{\partial F}{\partial \lambda}=\frac{E(\varphi ; \lambda)}{2 \lambda(1-\lambda)}-\frac{F(\varphi ; \lambda)}{2 \lambda}-\frac{\sin \varphi \cos \varphi}{2(1-\lambda) \sqrt{1-\lambda^{2} \sin ^{2} \varphi}}, \\
& \frac{\partial K}{\partial \lambda}=\frac{E(\pi / 2 ; \lambda)}{2 \lambda(1-\lambda)}-\frac{K(\lambda)}{2 \lambda},
\end{aligned}
$$

where $F(\varphi ; \lambda)$ and $K(\lambda)$ are as above and $E(\varphi ; \lambda)$ is an elliptic integral of the second kind,

$$
E(\varphi ; \lambda)=\int_{0}^{\varphi} \sqrt{1-\lambda \sin ^{2} \theta} d \theta .
$$

All these derivatives exist and are continuous for $\lambda \in(0,1)$, and so from the chain rule we obtain the lemma.

Lemma 1. $\rho(\mu, \nu) \in C^{1}(\mathcal{T})$.

Remark. The lemma says in particular that $\rho$ is continuous on $\mathcal{T}$, which allows us to answer the question raised in Example 2. Pick any point on the line $v=2-3 \mu$, say, $\mu=\frac{7}{12}$ and $\nu=\frac{1}{4}$. Then $\lambda=\frac{343}{375}, \cos \varphi=\frac{2}{7}$ and integration gives $\rho=\frac{1}{3}$. We are then able to conclude (by continuity) that $\rho=\frac{1}{3}$ along $\nu=2-3 \mu$ in $\mathcal{T}$. This illustrates the algorithmic utility of Lemma 1.

Beyond the smoothness of $\rho$ on $\mathcal{T}$, it is also important to understand what happens as we approach the boundaries of the parameter region $\mathcal{T}$. This knowledge will enable us to prove that in fact $\rho$ attains all values in $(0,1)$. First we examine the limiting behavior of $\rho(\mu, v)$ as we approach $(\mu, 0)$, where $\mu \in(0,1)$, from above (i.e., as we approach via a path in $\mathcal{T}$ ). Along any such path we have $\lambda \rightarrow 0^{+}$, and also the estimate,

$$
\varphi=\int_{0}^{\varphi} \frac{d \theta}{1} \leq \int_{0}^{\varphi} \frac{d \theta}{\sqrt{1-\lambda \sin ^{2} \theta}} \leq \int_{0}^{\varphi} \frac{d \theta}{\sqrt{1-\lambda}}=\frac{\varphi}{\sqrt{1-\lambda}} .
$$

Therefore $\rho(\mu, v) \rightarrow(1 / \pi) \varphi\left(\mu_{0}, 0\right)$ as $(\mu, v) \rightarrow\left(\mu_{0}, 0\right)$ in $\mathcal{T}$. This has a particularly simple form as we show next.

Lemma 2. As $(\mu, \nu) \rightarrow\left(\mu_{0}, 0\right)$ in $\mathcal{T}$, where $\mu_{0} \in(0,1)$, we have that

$$
\rho(\mu, \nu) \rightarrow \frac{2}{\pi} \arcsin \left(\frac{\mu_{0}}{2-\mu_{0}}\right) \text {. }
$$


Proof. We have $\rho(\mu, v) \rightarrow(1 / \pi) \varphi\left(\mu_{0}, 0\right)$. Now

$$
\frac{1}{\pi} \varphi\left(\mu_{0}, 0\right)=\frac{1}{\pi} \arccos \left(\frac{4-4 \mu_{0}-\mu_{0}^{2}}{\left(2-\mu_{0}\right)^{2}}\right)
$$

and if $r$ satisfies $(\sin \pi r) / 2=\mu_{0} /\left(2-\mu_{0}\right)$, then from familiar identities we get $\mu_{0}^{2} /$ $\left(2-\mu_{0}\right)^{2}=(1-\cos \pi r) / 2$ which implies that $\cos \pi r=\left(4-4 \mu_{0}-\mu_{0}^{2}\right) /\left(2-\mu_{0}\right)^{2}$. The result follows by putting $r=(1 / \pi) \varphi\left(\mu_{0}, 0\right)$.

Corollary 1. The level set in $\mathcal{T}$ corresponding to $\rho(\mu, \nu)=c$ approaches a unique point $(\mu(c), 0)$ on the $\mu$-axis (provided such a set approaches the $\mu$-axis at all).

Proof. The function $x /(2-x)$ increases strictly from 0 to 1 for $x \in[0,1]$.

There is an interesting connection between the limiting values of the rotation number $\rho(\mu, \nu)$ as we approach the base of the triangle $\mathcal{T}$ and the geometry of folding corresponding to $v=0$. On the $\mu$-axis the initial "trapezoid" is an isosceles triangle with its apex at $v_{1}=v_{2}$. Folding is still defined: the apex is fixed and $\pi^{2}$ acts as a rotation about this point by the apex angle $\alpha$. Now, the triangle at $\left(\mu_{0}, 0\right)$ has $\alpha$ given by $\alpha / 2 \pi=(1 / \pi) \arcsin \left(\mu_{0} /\left(1-\mu_{0}\right)\right)$, and so the rotation number of this folding is precisely half of the corresponding limiting value of $\rho(\mu, v)$. Nevertheless the diagonal dynamics for folding the triangle are trivial: all diagonal lengths are fixed. It is therefore surprising that the information contained in the rotation number is essentially conserved as we pass to the degenerate case. (In fact, if instead of taking the ratio of $\alpha$ to $2 \pi$ (to determine the fraction of a full turn) we take the ratio of $\alpha$ to $\pi$ so as to determine the fraction of the maximum possible apex angle, we find full agreement.)

Our goal is to show that for each $\rho_{0} \in(0,1)$, the level set $\rho(\mu, v)=\rho_{0}$ defines a curve in $\mathcal{T}$. We proceed by looking at line segments parallel to the base of $\mathcal{T}$ (defined by $\left.v=v_{0} \in\left(0, \frac{1}{2}\right)\right)$. We will demonstrate that $\rho \rightarrow 0$ as we approach the left boundary of $\mathcal{T}$ (i.e., let $\mu \rightarrow v_{0}^{+}$) and that $\rho \rightarrow 1$ as we approach the right boundary $\left(\mu \rightarrow\left(1-v_{0}\right)^{-}\right)$. We will also show that $\partial \rho / \partial \mu$ is not identically zero on any subinterval of $\left(\nu_{0}, 1-\nu_{0}\right)$. In other words, we will prove the following lemmas:

Lemma 3. For fixed $\nu_{0} \in\left(0, \frac{1}{2}\right)$, the rotation number $\rho\left(\mu, \nu_{0}\right)$ attains every value in $(0,1)$ as $\mu$ varies in $\left(v_{0}, 1-v_{0}\right)$.

Lemma 4. For fixed $v_{0} \in\left(0, \frac{1}{2}\right), \rho\left(\mu, v_{0}\right)$ is not constant on any subinterval of $\left(\nu_{0}, 1-\nu_{0}\right)$.

Delaying the proofs of the lemmas, we now prove the following theorem.

Theorem 1. For each $\rho_{0} \in(0,1)$ there is a unique curve in $\mathcal{T}$ defined by $\rho(\mu, \nu)=\rho_{0}$. This curve segment approaches the apex of $\mathcal{T}$ at one end and the point $(2 \sin (\pi / \rho) /$ $(1+\sin (\pi \rho / 2), 0)$ on the base at the other end. Moreover, this curve may be expressed in the form $\mu=f(v)$. 
Proof. Lemmas 1, 3, and 4 tell us that for each $\rho_{0} \in(0,1)$, the condition $\rho(\mu, \nu)=\rho_{0}$ defines one or more curves, and Lemma 3 further tells us that their graphs are defined arbitrarily close to the apex and base of $\mathcal{T}$. Lemma 2 says that all the curves corresponding to $\rho_{0}$ must approach the same point on the base of $\mathcal{T}$. Inverting the relation in this lemma, we find that this point is actually $((2 \sin \pi \rho / 2) /(1+\sin \pi \rho / 2), 0)$.

Now if there is more than one arc of curve corresponding to $\rho_{0}$, then these enclose a region containing a set of the form $\left(\mu_{1}, \mu_{2}\right) \times\left\{v_{1}\right\}$. Since curves corresponding to different rotation numbers cannot intersect, every point in this set must make $\rho=\rho_{0}$. However, this contradicts Lemma 4 , so $\rho(\mu, v)=\rho_{0}$ must define a unique curve. Furthermore, on each segment defined by $v=v_{0} \in\left(0, \frac{1}{2}\right)$, each value of $\rho$ can be obtained only once. In other words, the curves $\rho(\mu, \nu)=\rho_{0}$ pass the horizontal line test, and thus define $\mu$ as a function of $\nu$.

Remark. The apex of $\mathcal{T}$ (i.e., $\left.(\mu, \nu)=\left(\frac{1}{2}, \frac{1}{2}\right)\right)$ corresponds to the unit square. Theorem 1 tells us that for arbitrarily small pertubations from the square we can obtain the full range of diagonal dynamics (motion on the shoe $\Sigma$ ). More precisely, given any $\varepsilon>0$ and any $\rho_{0} \in(0,1)$, we can find $(\mu, v)$ so that $\left|(\mu, \nu)-\left(\frac{1}{2}, \frac{1}{2}\right)\right|<\varepsilon$ and so that the mapping $\tau$ on $\Sigma$ is conjugate to translation on $\mathbb{R} / 2 \pi \mathbb{Z}$ by the angle $2 \pi \rho_{0}$.

We now establish Lemmas 3 and 4.

Proof of Lemma 3. Fix $\nu \in\left(0, \frac{1}{2}\right)$. Recall that as $\mu \rightarrow v^{+}$, we have $\lambda \rightarrow 1^{-}$and $\varphi \rightarrow$ $\varphi_{0}^{+}$, where $\varphi_{0}=\varphi(v, v)=\arccos ((1-2 v) /(1-2 \nu+2 \nu))$. Then $\varphi_{0} \in(0, \pi / 2)$. Similarly, as $\mu \rightarrow(1-\nu)^{-}$, there follows $\lambda \rightarrow 1^{-}$and $\varphi \rightarrow \varphi_{1}^{-}$, where $\varphi_{1}=\varphi(1-\nu, \nu)=$ $\arccos \left((1-2 \nu)^{2} /\left(4 v^{2}-4 \nu-1\right)\right)$, and so $\varphi_{1} \in(\pi / 2, \pi)$. Recall also that $\varphi \in(0, \pi / 2)$ or $(\pi / 2, \pi)$ as $(\mu, \nu)$ is to the left or the right of the curve $\nu=\mu+2^{3 / 2} \sqrt{1-\mu}-2$.

So as $\mu \rightarrow v^{+}$, we have $\varphi<\pi / 2$, and $\varphi \rightarrow \varphi_{0}<\pi / 2$, and so $F(\varphi ; \lambda)$ remains bounded. On the other hand, $K(\lambda) \rightarrow \infty$ as $\lambda \rightarrow 1^{-}$, which implies that the denominator of $\rho$ increases without bound. (These are classical results. See, for example, [SO].) Hence $\rho \rightarrow 0^{+}$.

For the case $\mu \rightarrow(1-\nu)^{-}$, it is convenient to make use of the identity $F(\pi-\alpha ; \lambda)=$ $2 K(\lambda)-F(\alpha ; \lambda)$. Since $\varphi \in(\pi / 2, \pi)$ for $(\mu, \nu)$ to the right of $v=\mu+2^{3 / 2} \sqrt{1-\mu}-2$, we can take $\alpha=\pi-\varphi \in(0, \pi / 2)$ to conclude that $\alpha \rightarrow \alpha_{0}^{+}, \alpha_{0} \in(0, \pi / 2)$. Applying the above identity gives

$$
\rho(\mu, \nu)=\frac{2 K(\lambda)-F(\alpha ; \lambda)}{2 K(\lambda)}=1-\frac{F(\alpha ; \lambda)}{2 K(\lambda)} .
$$

The previous paragraph then shows that $\rho \rightarrow 1^{-}$. The result follows immediately from Lemma 1 and the intermediate value theorem.

Proof of Lemma 4. Fix $v \in\left(0, \frac{1}{2}\right)$. Suppose that $\rho$ is constant over $\left(\mu_{1}, \mu_{2}\right) \subseteq$ $(\nu, 1-\nu)$. Then $\partial \rho / \partial \mu=0$ for $\mu \in\left(\mu_{1}, \mu_{2}\right)$. The chain rule implies that

$$
\frac{\partial \rho}{\partial \mu}=\frac{1}{2} \frac{\partial}{\partial \mu}\left[\frac{F(\varphi ; \lambda)}{K(\lambda)}\right]
$$




$$
\begin{aligned}
& =\frac{1}{2} \cdot \frac{\left(K(\lambda)\left[\frac{\partial F}{\partial \varphi} \frac{\partial \varphi}{\partial \mu}+\frac{\partial F}{\partial \lambda} \frac{\partial \lambda}{\partial \mu}\right]-F(\varphi ; \lambda)\left[\frac{d K}{d \lambda} \frac{\partial \lambda}{\partial \mu}\right]\right)}{[K(\lambda)]^{2}} \\
& =\frac{1}{2[K(\lambda)]^{2}}\left[\frac{\partial \lambda}{\partial \mu}\left\{K(\lambda) \frac{\partial F}{\partial \lambda}-F(\varphi ; \lambda) \frac{d K}{d \lambda}\right\}+\frac{\partial \varphi}{\partial \mu}\left\{K(\lambda) \frac{\partial F}{\partial \varphi}\right\}\right] .
\end{aligned}
$$

Now by assumption, $\rho(\mu, v)=\rho_{0}$ (say) on $\left(\mu_{1}, \mu_{2}\right)$, so

$$
F(\varphi ; \lambda)=2 \rho_{0} K(\lambda)
$$

Taking the logarithmic derivative of both sides (both are strictly positive),

$$
\frac{(\partial F / \partial \lambda)(\varphi ; \lambda)}{F(\varphi ; \lambda)}=\frac{(d K / d \lambda)(\lambda)}{K(\lambda)},
$$

for $\mu \in\left(\mu_{1}, \mu_{2}\right)$. However, this implies that

$$
K(\lambda) \frac{\partial F}{\partial \lambda}(\varphi ; \lambda)-F(\varphi ; \lambda) \frac{d K}{d \lambda}(\lambda)=0
$$

on this interval. Combining all this gives the relation

$$
\begin{aligned}
0=\frac{\partial \rho}{\partial \mu} & =\frac{1}{2[K(\lambda)]^{2}}\left[\frac{\partial \lambda}{\partial \mu}\{0\}+\frac{\partial \varphi}{\partial \mu}\left\{K(\lambda) \frac{\partial F}{\partial \varphi}\right\}\right] \\
& =\left[\frac{\partial F}{\partial \varphi} \frac{\partial \varphi}{\partial \mu}\right] /[2 K(\lambda)]
\end{aligned}
$$

on $\left(\mu_{1}, \mu_{2}\right)$. However, neither $\partial F / \partial \varphi$ nor $\partial \varphi / \partial \mu$ are zero on an interval, so we have a contradiction.

Theorem 1 has the following corollary.

Corollary 2. Let $\mathbf{C}$ be any curve transversing $\mathcal{T}$ horizontally (i.e., crossing $\partial \mathcal{T}$ only at $\left.\left\{(\mu, v): \mu=\nu, v \in\left(0, \frac{1}{2}\right)\right\} \cup\left\{(\mu, v): \mu+v=1, v \in\left(0, \frac{1}{2}\right)\right\}\right)$. Then for each $\rho_{0} \in(0,1)$ there is a point on $\mathbf{C}$ at which $\tau$ is conjugate to rotation on a circle by $2 \pi \rho$.

Proof. Any such curve $\mathbf{C}$ intersects every curve defined by $\rho(\mu, v)=\rho_{0}$ at least once.

\section{Rational Dynamics on $\Sigma$}

In the previous section we used the fact that $\tau$ is translation on an elliptic curve to determine quite explicitly how this map behaves for various $(\mu, \nu) \in \mathcal{T}$. Given $\mu$ and $\nu$, we can-in theory-calculate $\rho(\mu, \nu)$, and hence determine if the $\tau$-orbit of the initial isosceles trapezoid is periodic (if $\rho=m / n \in \mathbb{Q}, \operatorname{gcd}(m, n)=1$, then $\tau^{n}=i d$ ) or dense on the shoe (this happens when $\rho \in \mathbb{Q}^{c}$ ). Unfortunately, calculating $\rho(\mu, \nu)$ can be very difficult to do explicitly. On the other hand, numerical evaluation of $\rho$ may not allow 
us to distinguish adequately between rational and irrational values: to a computer, all numbers are rational.

Fortunately, it is possible to use some other results on elliptic curves to obtain information about the diagonal dynamics with less computational difficulty.

Much of the work done on elliptic curves is number-theoretic, and so to make use of the most interesting results we restrict our attention to rational initial trapezoids, i.e., we take $(\mu, \nu) \in \mathcal{T} \cap \mathbb{Q}^{2}$. This allows us to apply the Nagell-Lutz and Mazur theorems (see $[S T])$.

Theorem 2. Let $(\mu, v) \in \mathcal{T} \cap \mathbb{Q}^{2}$. Suppose that $\mu=p / q, v=r / s$, with $\operatorname{gcd}(p, q)=$ $1=\operatorname{gcd}(r, s) . \operatorname{Set}$

$$
\begin{array}{r}
\sigma=\frac{2^{12} 3^{3} q^{9} s^{9} \mu \nu(1-\mu)(1-v)(1-\mu-\nu)(\mu+\nu)^{2}(\mu-\nu)^{2}(2-\mu-v)^{2}}{\left[\left(\mu^{2}+v^{2}+6 \mu \nu-4 \mu-4 \nu+4\right)\left(\mu^{2}+\nu^{2}-2 \mu \nu-4 \mu-4 v+4\right)\right.} \\
\left.\times\left(4-\mu^{2}-v^{2}+2 \mu \nu-4 \mu-4 \nu\right)\right]
\end{array}
$$

If $\sigma$ is undefined, then $\tau^{2}=i d$. If $\sigma$ is defined but is not an integer, then $\tau$ has infinite period and the $\tau$-orbit of the initial trapezoid is dense on the shoe. If $\sigma$ is an integer, then $\tau$ has infinite period if and only if $\tau^{j} \neq i$ d for any of $j=7,8,9,10,12$.

Proof. Putting $\mu=p / q, v=r / s$ into the expression defining $W_{\mu \nu}$ gives

$$
y^{2}=4 x^{3}+\frac{4 G_{2}}{27 q^{9} s^{9}} x+\frac{4 G_{3}}{27 q^{9} s^{9}},
$$

where $G_{2}, G_{3} \in \mathbb{Z}$. If we apply the transformation

$$
x \rightarrow \frac{x}{9 q^{6} s^{6}}, \quad y \rightarrow \frac{2 y}{27 q^{9} s^{9}},
$$

we get

$$
W_{\mu \nu}^{\prime}: \quad y^{2}=x^{3}+\left(3 q^{3} s^{3} G_{2}\right) x+27 q^{9} s^{9} G_{3} .
$$

The corresponding discriminant is

$$
\Delta^{\prime}=-27 q^{9} s^{9}\left[4 G_{2}^{3}+27^{2} q^{9} s^{9} G_{3}^{2}\right] .
$$

Applying the above transformation to $H=\left(H_{x}, H_{y}\right)=\left(\left(Q^{2}+8 L\right) / 12,-C\right)$ gives

$$
\begin{aligned}
& H_{x}^{\prime}=\frac{3 q^{6} s^{6}\left(Q^{2}+8 L\right)}{4}, \\
& H_{y}^{\prime}=\frac{-27 q^{9} s^{9} C}{2} .
\end{aligned}
$$

Now, the Nagell-Lutz theorem tells us that if $H$ has finite order, then $H_{x}^{\prime}, H_{y}^{\prime} \in \mathbb{Z}$, and either $H_{y}^{\prime}=0$ or $\left(H_{y}^{\prime}\right)^{2} \mid \Delta^{\prime}$. The denominator of $\sigma$ is zero in $T$ precisely along $\nu=\mu+2^{3 / 2} \sqrt{1-\mu}-2$ (defined by $C=0$ ). As seen above, this curve corresponds to $\rho=\frac{1}{2}$, so that $\tau^{2}=i d$ here. 
Explicit computation of $H_{x}^{\prime}$ shows that it is automatically an integer under the assumptions of the theorem. A similar computation gives that

$$
\frac{\Delta^{\prime}}{\left(H_{y}^{\prime}\right)^{2}}=\sigma^{2} \text {. }
$$

Since $\sigma \in \mathbb{Q}$, it is clear that $\sigma^{2} \in \mathbb{Z}$ if and only if $\sigma \in \mathbb{Z}$. Moreover, if $\sigma \in \mathbb{Q} \backslash \mathbb{Z}$, then $H$ cannot have finite order and so $\tau$ has infinite period.

If $\sigma \in \mathbb{Z}, H$ may or may not have finite order. Mazur's theorem says that the subgroup of rational points of $W_{\mu \nu}$ having finite order is isomorphic to $C_{n}$ for $n=1, \ldots, 10,12$ or $C_{2} \oplus C_{2 m}$ for $m=1, \ldots, 4$. Lagrange's theorem then gives that the order of any such point must divide one of $7,8,9,10,12$, in which case $\tau^{j}=i d$ for the appropriate value of $j$.

Remark. If $\rho_{0}=p / q, \operatorname{gcd}(p, q)=1, q>12$, then this says that the curve defined by $\rho(\mu, \nu)=\rho_{0}$ contains no rational points.

Corollary 3. Let $(\mu, v) \in \mathcal{T} \cap \mathbb{Q}^{2}$. Then either $\pi^{n}$ acts as a rigid motion on the initial trapezoid for some $n$ with $1 \leq n \leq 48$, or else the original trapezoid never recurs (in any orientation).

Proof. By Theorem 2, either $H$ has infinite order or has order no more than 12 . In the latter case, a quadrilateral congruent to the original (possibly with orientation reversed) occurs on or before the 24th folding. Doubling the maximum number of iterations ensures that the orientation is preserved, and hence gives the result.

\section{The Simplest Singular Map of the Plane}

Consider the following family of maps defined on $\mathbb{R}^{2} \backslash(\{0\} \times \mathbb{R})$ :

$$
T_{\alpha}(x, y)=\left(y, \frac{y+\alpha}{x}\right)
$$

(where $\alpha \in \mathbb{R}$ ). If we fix $\alpha$ and pick some initial point $\left(x_{0}, y_{0}\right)$ not on either axis, then it is easy to verify that $T_{\alpha}$ leaves invariant the curve defined by

$$
\Gamma_{\alpha \beta}: \quad \beta x y=(x+1)(y+1)(x+y+\alpha),
$$

where $\beta$ is defined by

$$
\beta=\frac{\left(x_{0}+1\right)\left(y_{0}+1\right)\left(x_{0}+y_{0}+\alpha\right)}{x_{0} y_{0}},
$$

and so $\beta$ is uniquely defined by the initial conditions.

For most values of $\alpha$ and $\beta, \Gamma_{\alpha \beta}$ is a real elliptic curve. Indeed, if $\beta \neq 0$ and $\alpha \neq \beta+1$, then the following series of transformations puts $\Gamma_{\alpha \beta}$ into pre-Weierstrass 
(i.e., $y^{2}=x^{3}+a x^{2}+b x+c$ ) form:

(a) $x \rightarrow(1+(\beta-\alpha) x+y) / 2 x, y \rightarrow(1+(\beta-\alpha) x-y) / 2 x$.

(b) $x \rightarrow x, y^{2} \rightarrow 4 \beta(\beta-\alpha+1) y^{2}$.

One can then calculate the discriminant and determine precisely when $\Gamma_{\alpha \beta}$ fails to be an elliptic curve.

The family $T_{\alpha}$ is in some way the "simplest family" of singular maps of the plane: it is singular only along $x=0$, and half of the action of the map is just a shift.

It turns out that there is an interesting connection between $\left(T_{\alpha}, \Gamma_{\alpha \beta}\right)$ and $\left(\tau, X_{\mu \nu}\right)$. Pick a point in parameter space $\mathcal{T}$ satisfying $C \neq 0$ and $L \neq 0$. (Recall that $C=0$ corresponds to $2 H=O$, and $L=0$ corresponds to $3 H=O$. In both cases we know the full story about both $\tau$ and $\pi$.) Define the projective transformation $\eta$ by

$$
\eta:\left\{\begin{array}{l}
X^{\prime}=L X-C Z, \\
Y^{\prime}=L Y-C Z, \\
Z^{\prime}=C Z .
\end{array}\right.
$$

Elementary algebra then establishes the following.

Proposition 2. Suppose $C L \neq 0$ and $\eta$ is as defined above. Then $\eta$ carries the projective completion of $X_{\mu \nu}$ bijectively to the projective completion of $\Gamma_{\alpha \beta}$, where

$$
\begin{aligned}
& \alpha=2-\frac{L}{C}\left(Q+\frac{L^{2}}{C}\right), \\
& \beta=-\frac{L^{3}}{C^{2}} .
\end{aligned}
$$

Remark. Since $\eta$ is nonsingular, the resulting $\Gamma_{\alpha \beta}$ is elliptic.

As mentioned previously, a map such as $\eta$ is actually a group isomorphism from $X_{\mu \nu}$ to $\Gamma_{\alpha \beta}$. Thus, $\tau: P \mapsto P+h$ is equivalent to some map $f: \eta(P) \mapsto \eta(P+h)=$ $\eta(P)+\eta(h)$, i.e., to translation by $\eta(h)$. A simple calculation shows that

$$
\eta(h)=\eta([0, C, L])=[-C L, 0, C L]=[-1,0,1]=(-1,0) .
$$

So $\tau$ is equivalent to translation on $\Gamma_{\alpha \beta}$ by the point $(-1,0)$. What is more, translating by $(-1,0)$ is the same as applying $T_{\alpha}^{2}$.

Theorem 3. If $C L \neq 0$ and $T_{\alpha}^{2}$ is defined, then the following diagram commutes:

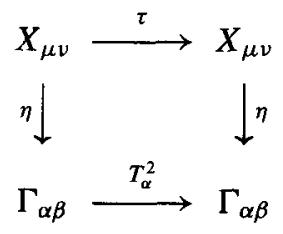

Proof. By the above discussion, it remains only to show that for a point $P=(x, y)$ on the shoe $\Sigma$ with $\eta(P)=(p, q), p q \neq 0$ (so that $T_{\alpha}^{2}$ is defined), we have $T_{\alpha}^{2}(\eta(P))=$ $\eta(P)+(-1,0)$ (where the addition is on the elliptic curve $\Gamma_{\alpha \beta}$ ). 
Notice that $p=-1 \Longleftrightarrow x=0$, and $p+q+1=0 \Longleftrightarrow x+y=C / L$. Recall that the shoe is caged by $x=0, y=0, x+y=Q(Q>0)$. Thus $p=-1$ never occurs. Also, putting $x+y=C / L$ in the definition of $X_{\mu \nu}$ gives $x y(x+y-Q)=0$, so we see also that $p+q+1=0$ never occurs.

Now, the line through $(p, q)$ and $(-1,0)$ is given by

$$
\ell: \quad y^{\prime}=\left(\frac{q}{p+1}\right)\left(x^{\prime}+1\right) .
$$

If we put $\zeta\left(x^{\prime}, y^{\prime}\right)=\left(x^{\prime}+1\right)\left(y^{\prime}+1\right)\left(x^{\prime}+y^{\prime}+\alpha\right)-\beta x^{\prime} y^{\prime}$, then to solve for the third intersection of $\ell$ and $\Gamma_{\alpha \beta}$ we make the appropriate substitution and set $\zeta\left(x^{\prime}, y^{\prime}\right)=0$ :

$$
0=\zeta\left(x^{\prime}, y^{\prime}\right)=\frac{(p+q+1)\left(x^{\prime}+1\right)\left(x^{\prime}-p\right)\left(p q x^{\prime}-\alpha p-q-\alpha\right)}{(p+1)^{2} p}
$$

Factoring out our initial points, we get

$$
0=\frac{(p+q+1)\left(p q x^{\prime}-\alpha p-q-\alpha\right)}{(p+1)^{2} p} .
$$

By the above, this implies that

$$
0=p q x^{\prime}-\alpha p-q-\alpha
$$

so that the third intersection is given by

$$
\left(\frac{\alpha p+q+\alpha}{p q}, \frac{q+\alpha}{p}\right) .
$$

Now, $\eta([1,-1,0])=[1,-1,0]$, so the appropriate zero for $\Gamma_{\alpha \beta}$ is also $[1,-1,0]$. Hence we must reflect the above point in the line $y=x$ to get $\eta(P)+(-1,0)$ :

$$
\eta(P)+(-1,0)=\left(\frac{q+\alpha}{p}, \frac{\alpha p+q+\alpha}{p q}\right) .
$$

However, a simple calculation shows that

$$
T_{\alpha}^{2}(p, q)=\left(\frac{q+\alpha}{p}, \frac{\alpha p+q+\alpha}{p q}\right)
$$

as desired.

Remark. Although it may seem strange that $\tau$ is conjugate to a singular map, recall that the duplication formula for points on an elliptic curve in Weierstrass form is undefined when $2 P=O$.

We can use the form of $T_{\alpha}^{2}$ to obtain an explicit form for $\tau$. 
Corollary 4. Let $P=(x, y)$ be a point on the shoe $\Sigma$ with $x \neq C / L, y \neq C / L$, and $C L \neq 0$. Then

$$
\tau(P)=\left(\frac{C x+C y-Q C+L^{2}}{L x-C}, \frac{x\left(C L y+C^{2}-Q L C-L^{3}\right)}{(L x-C)(L y-C)}\right) .
$$

Proof. By assumption Theorem 3 applies and gives

$$
\tau(P)=\left(\eta^{-1} \circ T_{\alpha}^{2} \circ \eta\right)(P) .
$$

A direct application of this composite map gives the result.

\section{Conclusions}

The fact that $\tau$ is actually translation on an elliptic curve has proven to be very useful. It immediately gives that $\tau$ is analytically isomorphic to the simplest nontrivial map of the circle (rotation by $2 \pi \rho$ ); moreover, classical results on elliptic integrals have provided the machinery to study $\rho(\mu, \nu)$ quite fully (albeit qualitatively). For the case of rational $\mu$ and $\nu$, a little number theory allowed the development of a complete and relatively convenient algorithm for determining the dynamical behavior of $\tau$. Some of this information could in fact be "lifted" to $\pi$ in order to prove a surprising upper bound on its possible periods (in the case of rational $\mu$ and $\nu$ ).

As with many dynamical systems, the rationality or irrationality of certain parameters/ ratios seems to play a critical role in determining the dynamics of quadrilateral folding. In some instances (e.g., $\rho(\mu, v) \in \mathbb{Q}^{c}$ ) this duality is well understood. However, it is not clear at this point if the dynamical results obtained for $\mu, \nu \in \mathbb{Q}$ (specifically, Corollary 3) are valid only for rational values of the parameters. The methods applied were essentially number-theoretic, and so they do not carry over to the case $\mu, v \in \mathbb{Q}^{c}$ in a straightforward manner. Despite this, the fact that we are talking about the dynamics of a "physical" system (i.e., quadrilaterals) somehow suggests that the rationality of the parameters themselves should not be that important. Further work may allow the extension of some of these results to the case of irrational $\mu$ and $v$.

It would also be interesting to look more generally at dynamical systems defined by parameterized families of translations on abelian groups other than elliptic curves. Alternatively, one could try to characterize two-dimensional maps which have elliptic curves as their invariant manifolds. Under what conditions are such maps equivalent to translation by a point on the curve?

As for the problem of quadrilateral folding, several questions still remain. As mentioned above, one would like to know how important the rationality of $\mu$ and $\nu$ really is. Also, the question which initially motivated our study of quadrilateral folding - namely the question of predicting whether a given $(\mu, \nu)$-polygraph is bounded or unboundedremains open. Numerical evidence suggests that all such graphs are unbounded for $\mu+\nu>1$, but as yet there is no explanation for this. Computer plots also show symmetries which cannot yet be accounted for.

It would also be interesting to exploit further the connection between $\tau$ and $T_{\alpha}$. The simplicity of the latter map invites the application of the conventional methods of dynamical systems, e.g., linear analysis about the (at most) two equilibrium points. For 
example, there is a saddle-center bifurcation at $\alpha=\frac{3}{4}$ (a saddle-center pair bifurcates to a center-center pair as $\alpha$ increases through $\frac{3}{4}$ ). The task of verifying analytically that a neutrally stable equilibrium is a dynamical center is resolved algebraically here: the nested invariant curves surrounding an equilibrium are, of course, the closed components of our two-parameter family of elliptic curves. Similar investigations of the bifurcation structure of the map has revealed no new significant information; there is no avoiding the fact that a serious study requires the machinery of elliptic functions, and a further explication of the mysteries of polypath dynamics (e.g., the local loop structures) will no doubt require original methods of an intrinsically geometric nature.

\section{Final Remarks}

\section{Some History (Rogers)}

The bulk of the previous text was composed during the summer of 1999 . In the spring of 2000 we became aware that the map $T_{\alpha}$ which is the subject of Section 4 has been studied in some detail by other mathematicians, most notably Sir E. Christopher Zeeman. The interested reader is directed to Professor Zeeman's lecture given as part of the Distinguished Lecturer Series at the Pacific Institute of Mathematical Sciences [Z3], as well as the papers $[\mathrm{Z1}]$ and $[\mathrm{Z2}]$. At the time of writing these final remarks we had access only to the filmed version of Zeeman's work. (See also [BC] and [S1] in the references.)

In the sources mentioned above, Zeeman studies $T_{\alpha}$ as the "geometrical unfolding" of a difference equation which he calls the generalized Lyness equation: $x_{n+1}=\left(x_{n}+\right.$ $\alpha) / x_{n-1}, \alpha>0$. James Lyness (of Argonne Labs) has explained to me that his father, R.C. Lyness, was an amateur mathematician who did some "very elegant stuff about recurring sequences which was ignored in his lifetime" [L].

My own adventures with $T_{\alpha}$ began independently in the $80 \mathrm{~s}$. I was very interested in studying noninvertible planar maps which were "the simplest of their type." Motivated entirely by esthetic considerations, I was looking for simply defined maps which exhibited rich dynamics. Having studied polynomial maps, I decided to write down the simplest rational map whose asymptotic properties were not immediately obvious. Thus I was led to consider $T_{\alpha}$.

While we were collaborating in 1984, James Pounder noticed the existence of the invariant cubic curves $\Gamma_{\alpha \beta}$. Between then and 1990 (at which point I summarized my work done with Pounder for a grant application to NSERC) I obtained various results about $T_{\alpha}$. To be brief, I studied the fixed points of the map and their bifurcations (under variation of $\alpha$ ), related the action of the map to translation under the group law on the real elliptic curves $\Gamma_{\alpha \beta}$, and showed that $T_{\alpha}$ is conjugate to translation on a circle.

In 1991 I came across some exercises in the most recent edition of [NZM] (p. 280, Exercises 18 and 19) related to $T_{\alpha}$ and its variant elliptic curves. When I contacted Montgomery about this, he said that he had learned of the map from John Conway.

I first began studying polypaths in 1986 after seeing some computer-generated images created by Ted Lewis. The connections between polypaths and $T_{\alpha}$ did not come to light until the early 90s. By the summer of 1996 I was giving talks on these connections at Brian Alspach's discrete geometry seminar at Simon Fraser University. 


\section{Comparison of Results}

Here we wish to give a brief comparison of our results with those obtained independently by Zeeman and others.

Zeeman begins by finding a Lyapunov function for $T_{\alpha}$ which is in fact the invariant quantity we call $\beta$. Next he uses the fact that the level curves of this Lyapunov function are real elliptic curves to connect $T_{\alpha}$ to rotation on a circle. This of course follows from Theorem 3 of this paper. He uses this result to obtain bounds on the rotation number of the map when $\alpha$ is constrained to lie in certain intervals. He conjectures that the rotation number is a monotonic function within each of these $\alpha$-intervals, but his geometrical methods do not allow him to prove it. Beukers and Cushman [BC] prove this result by making use of the Weierstrass $\wp$-function. The spirit of their work is quite similar to Section 2 of this paper. In fact, Theorem 1 can be viewed as an analogue of "Zeeman's monotonicity conjecture" in the polypath setting, and we suspect that it can be used to give an alternate proof of the result of [BC].

Using classical number theory (in particular the Prime Number Theorem and Chebyshev's Inequality), Zeeman derives the exact list of possible periods of $T_{\alpha}$. This list is infinite, and in fact includes all sufficiently large integers. On the other hand, Corollary 3 of this paper gives a finite list of possible periods of $\pi$ for rational values of $\mu$ and $\nu$. Our result thus shows that for all sufficiently large periods of $T_{\alpha}$, the values of $\alpha$ and $\beta$ giving these periods must correspond to irrational values of $\mu$ and $\nu$. (Compare this with the Remark following Theorem 2.)

As a final comparison, we note that Zeeman derives a formula (depending on the value of $\alpha$ under consideration) for the limiting value of the rotation number as $\beta$ approaches its minimum. It is arcsine of a rational function of $\alpha$ and is the analogue of Lemma 2 in the context of $T_{\alpha}$.

In principle, Proposition 2 and Theorem 3 provide a way to translate every statement about $\tau$ to a statement about $T_{\alpha}$, and vice versa. Unfortunately, $\alpha$ and $\beta$ are fairly complex rational functions of $\mu$ and $\nu$, and this makes it difficult to determine ranges of $\mu$ and $\nu$ for given ranges of $\alpha$ and $\beta$. This relationship would have to be unraveled in order to use Theorem 1 to prove the result of [BC], or (going the other way) to use Zeeman's list of possible periods to extend Corollary 3 to the case of irrational $\mu$ and $\nu$. Maple may be of some use here.

\section{Acknowledgment}

We wish to thank an anonymous referee for suggesting some useful clarifications in our exposition.

\section{Appendix. Polypath Program}

Maple $\mathrm{V}$ code for plotting polypaths. The input parameters are $\mu \in(0,1), v \in\left(0, \frac{1}{2}\right)$, and $n \in \mathbb{N}$. $\mu$ and $v$ define the side lengths of an initial isosceles trapezoid. $n$ determines 
how many points will be plotted for each of the forward and backward orbits (so $n$ gives $2 n$ points in (otal).

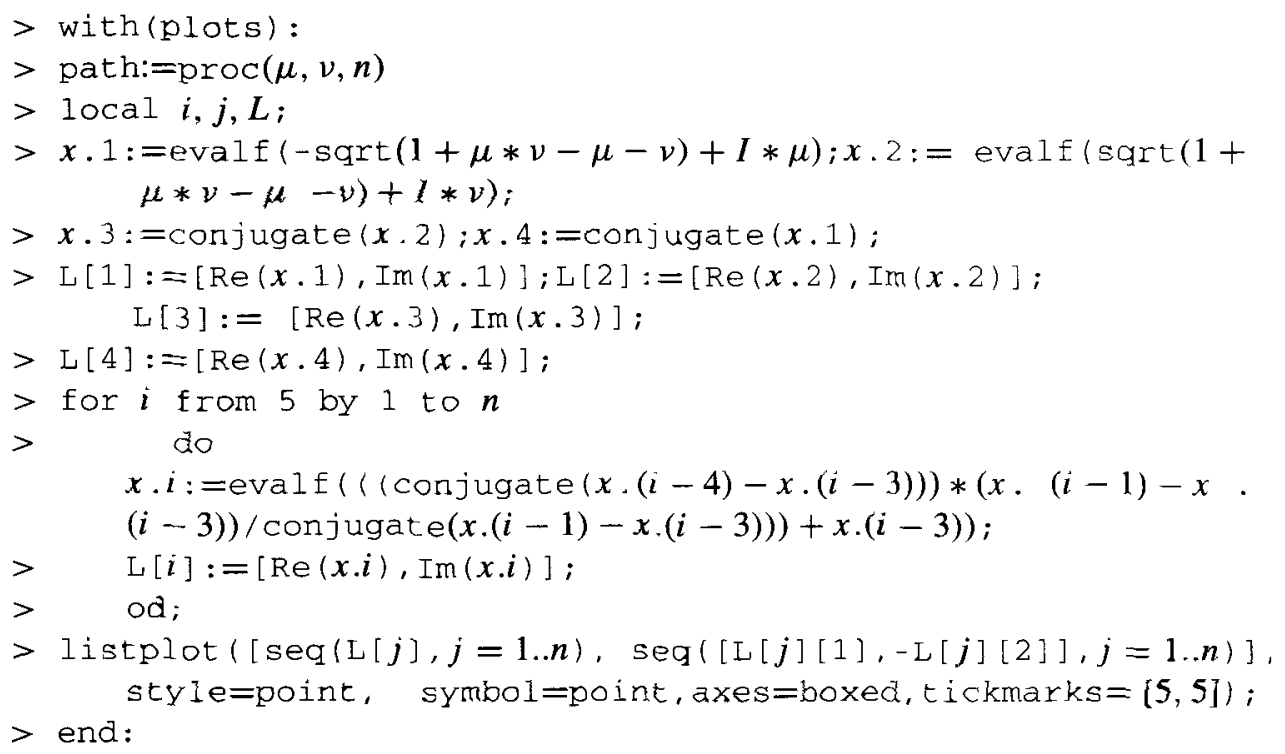

\section{References}

[AS] Milton Abraham and Irene A. Stegun, editors, Handbook of Mathematical Functions with Formulas, Graphs and Mathematical Tables, Dover, New York, 1965.

[BC] Frits Beukers and Richard Cushman, Zeeman's Monotonicity Conjecture, Journal of Differential Equations 143 (1998), 19l-200.

[CR] Kevin Charter and Thomas Rogers, The Dynamics of Quadrilateral Folding, Experimental Mathematics 2 (1993), 209-222.

[D] Robert L. Devaney, An Introduction to Chaotic Dynamical Systems, second edition, Addison-Wesley, Redwood City, CA, 1989

[K] Neal Koblitz, Introduction to Elliptic Curves and Modular Forms, second edition, Springer-Verlag, New York, 1993.

[L] James Lyness, Personal communication, May 2000.

[M] Ricardo Mané, Ergodic Theory and Differential Dynamics, Springer-Verlag, New York, 1987.

[MM] Henry McKean and Victor Moll, Elliptic Curves, Cambridge University Press, Cambridge, 1997.

[NZM] lvan Nivin, Herbert S. Zuckeman, and Hugh L. Montgomery, An Introduction to Theory of Numbers, fifth edition, Wiley, New York, 1991.

[S1] Michal Sierakowski, A Generalization of Zeeman's Family, Fundamenta Mathematicae 162 (1999), 277-286.

[S2] Joseph A. Silverman, The Arithmetic of Elliptic Curves, Springer-Verlag, New York, 1986.

[SO] Jerome Spanier and Keith B. Oldman, An Atlas of Functions, Hemisphere, Washington, DC, Harper and Row. Berlin, 1987.

[ST] Joseph H. Silverman and John Tate, Rational Points on Elliptic Curves, Springer-Verlag, New York, 1992.

[Z1] Christopher Zeeman, A Geometric Unfolding of a Difference Equation, Journal of Difference Equations and Applications, to appear. 
[Z2] Christopher Zeeman, Higher Dimensional Unfoldings of Difference Equations, Lecture Notes, ICTP Conference, Trieste, September 1998.

[Z3] Christopher Zeeman, Lyness Equation, Distinguished Lecture Series, Pacific Institute of Mathematic Sciences, 2000. See recorded film at web site http://www.pims.math.ca/activities/dist.lect/zeeman/ zeeman.html.

Received October 21, 1999, and in revised form April 13, 2000 and May 24, 2000.

Online publication September 22, 2000. 